\title{
Is white rice consumption a risk for metabolic and cardiovascular outcomes? A systematic review and meta-analysis
}

\author{
Chayakrit Krittanawong, ${ }^{1,2}$ Anusith Tunhasiriwet, ${ }^{2}$ HongJu Zhang, ${ }^{2}$ Larry J Prokop, ${ }^{3}$ \\ Sakkarin Chirapongsathorn, ${ }^{4,5}$ Tao Sun, ${ }^{2}$ Zhen Wang ${ }^{6,7}$
}

- Additional material is published online only. To view please visit the journal online (http://dx.doi.org/10.1136/ heartasia-2017-010909).

${ }^{1}$ Department of Internal Medicine, Icahn School of Medicine at Mount Sinai St' Luke and Mount Sinai West, New York, New York, USA ${ }^{2}$ Division of Cardiovascular Diseases, Department of Medicine, Mayo Clinic, Rochester, Minnesota, USA ${ }^{3}$ Mayo Clinic Libraries, Rochester, Minnesota, USA

${ }^{4}$ Division of Gastroenterology and Hepatology, Department of Medicine, Mayo Clinic, Rochester, Minnesota, USA ${ }^{5}$ Division of Gastroenterology, Phramongkutklao Hospital and College of Medicine, Royal Thai Army, Bangkok, Thailand

${ }^{6}$ Robert D. and Patricia E. Kern Center for the Science of Health Care Delivery, Rochester, Minnesota, USA

${ }^{7}$ Division of Health Care Policy and Research, Department of Health Sciences Research, Mayo Clinic, Rochester, Minnesota, USA

Correspondence to Dr Chayakrit Krittanawong, Department of Internal

Medicine, Icahn School of Medicine at Mount Sinai St' Luke and Mount Sinai West, Tenth Avenue, Suite 3A-09, New York 10019 NY, USA; chayakrit. krittanawong@mountsinai.org

Received 21 May 2017 Revised 25 July 2017

Accepted 26 July 2017

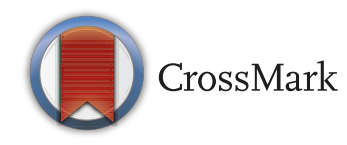

\footnotetext{
To cite: Krittanawong $C$, Tunhasiriwet $\mathrm{A}$, Zhang $\mathrm{HJ}$, et al. Heart Asia

2017:9:1-7.. doi:10.1136/ heartasia-2017-010909
}

\begin{abstract}
Objective The main objective of this systematic review and meta-analysis was to investigate the association between white rice consumption and risk of metabolic and cardiovascular outcomes.

Methods We conducted a comprehensive search of Medline, Embase, Scopus, and the Cochrane Central Register of Controlled Trials from database inception through March 2016. Original studies that reported

To date, previous meta-analyses have not found an association between rice intake and cardiovascular outcomes. ${ }^{9}$ We hypothesised that white rice consumption is not associated with metabolic and cardiovascular outcomes. Therefore, we performed a systematic review and meta-analysis on all published studies to date evaluating the association between white rice consumption and cardiovascular outcomes, including T2DM.
\end{abstract} associations between white rice consumption and cardiovascular outcomes regardless of study design were selected. We extracted study characteristics and outcome data. Conflicts were resolved through consensus. Using the DerSimonian and Laird random effects models, we calculated pooled relative risks with $95 \% \mathrm{Cl}$.

Results Our search identified 721 citations. 18 studies were included with a total of 1777059 individuals: 14348 had type 2 diabetes mellitus (T2DM); 5612 had metabolic syndrome (MetS); 10839 had coronary heart disease (CHD); and 11698 had stroke. Compared with the lowest category, the highest category of white rice consumption was only associated with $30 \%$ higher risk of MetS (pooled OR 1.30, 95\% Cl 1.03 to 1.65; $\left.p<0.001 ;\left.\right|^{2}=65.5 \%\right)$.

Conclusions Higher white rice consumption has not been shown to be associated with increased risk of CHD, stroke and T2DM. However, white rice consumption may be associated with increased risk of MetS in certain populations

\section{INTRODUCTION}

The current Dietary Guidelines for Americans 20152020 advocate replacing refined grains (eg, white rice) with whole grains (eg, brown rice) for improved health outcomes. ${ }^{1}$ However, less is known about the cardiovascular effects among types of rice (brown rice vs white rice). Brown rice contains high natural nutrition (eg, phosphorus, magnesium, iron, folic acid, and $B$ vitamins), fibre, and antioxidants (eg, selenium and manganese) and has been shown to be associated with lower inflammatory marker levels, blood glucose in type two diabetes mellitus (T2DM), and obesity. ${ }^{2}$ White rice, in contrast, has lower nutritional content and a higher glycaemic index which may be associated with an increased risk of developing T2DM. ${ }^{4}$ Further, preliminary studies report that replacing white rice with brown rice may lower the risk of T2DM. $^{5}$ However, additional studies ${ }^{6-8}$ report no relationship between white rice consumption and T2DM, and therefore the relationship between white rice consumption and T2DM remains controversial.

\section{METHODS}

\section{Search strategy}

A comprehensive search of five databases from 1966 to 3 March 2016, in any language and involving humans only, was conducted. The databases included Ovid Medline In-Process \& Other Non-Indexed Citations, Ovid Medline, Ovid Embase, Ovid Cochrane Central Register of Controlled Trials, and Scopus. The search strategy was designed and conducted by two authors (CK and LJP). Controlled vocabulary supplemented with keywords was used to search for studies on white rice consumption and risk of metabolic outcomes (T2DM and metabolic syndrome (MetS)) and cardiovascular outcomes (coronary heart disease (CHD), acute myocardial infarction, coronary artery disease, ischaemic heart disease, acute coronary syndrome, sudden cardiac arrest, ischaemic or haemorrhagic stroke). The full search strategies can be found in the online supplement (supplementary emethods).

\section{Study selection}

Studies were included in this meta-analysis if they satisfied the following criteria: the study design was prospective, cross-sectional, case-control, randomised or non-randomised control trial, and the exposure of interest was white rice consumption. The outcomes were T2DM, MetS, CHD or stroke. Reviews, editorials, non-human studies, and letters without sufficient data were excluded.

\section{Data extraction} two authors (CK and AT) using a standard extraction form. We extracted the following information from each study: authors, year of publication, study name, study location, years of follow-up, sample size (number of participants and incident cases), diagnostic criteria, participants' characteristics (age, sex and body mass index), endpoints (T2DM, MetS, CHD, and stroke), exposure and outcomes ascertainment, white rice consumption
Data extraction was carried out independently by 
categories, covariates adjusted in the multivariable analysis, relative risks (RRs), odd ratios (ORs), hazard ratios (HRs) and their 95\% confidence intervals (CIs) for all categories of white rice consumption (eTable 4). We contacted the authors if the data of interest were not reported in the manuscripts. We also consulted dietitians and nutritionists for the reliability and reproducibility of validation of all food frequency questionnaires (FFQ).

\section{Quality assessment}

Two authors (CK and AT) independently assessed the quality of included studies by using the modified Newcastle-Ottawa Scale. ${ }^{10}$ We resolved disagreements by discussion or involving the co-authors (TS, SC or HZ) to adjudicate and establish consensus. This scale awards a maximum of nine points to each study and eight points for a cross-sectional study (eTable 1-3). We assigned scores of $0-3,3.5-6$, and 6.5-9 for low, moderate, and high quality of studies for case-control and prospective studies, respectively, while scores of $0-2,2.5-5$, and 5.5-8 were assigned for low, moderate, and high quality cross-sectional studies.

\section{Statistical analysis}

We extracted or calculated the RR and 95\% CI from the included studies. For studies reporting OR or HR, because cardiovascular events were rare (incidence $<10 \%$ ), an asymptotically approach of $\mathrm{HR}$ and $\mathrm{OR}$ were treated as RR in the analysis. We converted RR to $\log$ transformed RR ( $\log R R)$ and pooled the $\log R R$ from all of the included studies using the DerSimonian and Laird random effects method ${ }^{11}$ with the estimate of heterogeneity from the Mantel-Haenszel model. ${ }^{12}$ We conducted subgroup analyses stratified by age ( $<40 v s \geq 40$ years), sex (female vs male), diagnostic criteria (WHO vs International Classification of Diseases and Related Health Problems, 10th revision (ICD-10) vs International Diabetes Federation (IDF) vs National Cholesterol Education Program Adult Treatment Panel III (NCEP-ATP III)), follow-up years ( $<10$ vs $\geq 10$ years), geographic location (region and country), type of study (prospective, case-control or cross-sectional), type of exposure (FFQ vs interview), outcome assessments (T2DM, MetS, CHD, and stroke) and quality assessment of included studies. The performances of subgroup-specific and statistical test of interaction among subgroups were assessed. We used the 'leave-one-out' method to evaluate whether any one study had a dominant effect on outcomes. Heterogeneity between the studies was evaluated using $I^{2}$ in which $>50 \%$ suggest substantial heterogeneity. Due to the limited number of studies included in each analysis and/or substantial heterogeneity, we could not evaluate potential publication bias. All statistical analyses were performed with OpenMetaAnalyst for 64-bit (Brown University), R version 3.2.3 (Metafor and Phia packages) and Stata version 11 (Stata Corp, College Station, Texas), and all tests were two sided with a significance level of 0.05 . The meta-analysis has been reported in accordance with the Meta-analysis of Observational Studies in Epidemiology (MOOSE) guidelines . ${ }^{13}$

\section{RESULTS}

\section{Literature search}

The database searches between 1966 and 3 March 2016 yielded 721 references (figure 1); 12 duplicates were removed. We screened 684 titles and abstracts. After this screening, 18 observational studies met our inclusion criteria: four studies ${ }^{14-17}$ estimated the ORs or HRs in the context of CHD; five studies $^{14}{ }^{15}$ 17-19 $^{19}$ estimated the ORs or HRs in the context of stroke (ischaemic or haemorrhagic stroke); eight studies ${ }^{5-8} 20-23$ estimated the ORs or HRs in the context of T2DM; and five studies $^{2024-27}$ estimated the ORs or HRs in the context of MetS. The disposition of studies excluded after full-text review are shown in figure 1.

\section{Study characteristics}

table 1 shows the basic characteristics of the included studies. In total, our meta-analysis included 18 studies with a total of 1777059 individuals: 14348 cases hadT2DM; 5612 had MetS; 10839 had CHD; and 11698 had stroke. Among the participants, we documented follow-up periods ranging from 14-23 years for CHD, 1-23 years for stroke, and 4-22 years for T2DM. Eleven cohorts ${ }^{15} 171921$ were conducted in Japan, four cohorts ${ }^{25-27}$ were conducted in Korea, five cohorts ${ }^{16} 18$ were conducted in China, six cohorts ${ }^{14}$ were conducted in the USA, and the others ${ }^{20} 24$ were conducted worldwide (Hong Kong, Australia, Spain, and Iran). White rice consumption was measured by FFQ in 27 cohorts and five cohorts ${ }^{6182025}$ utilised the 24-hour recall method with direct interview. Results of study quality assessment (score 0-9) yielded a score of 6.5 or above (high quality) for 18 studies, with an average score of 7.4 (online supplementary etables 1 and 2). The patient characteristics of the included studies are listed in table $1^{8-1125-30}$ figures 2 and 3 present forest plots of RRs and 95\% CIs and heterogeneity statistics for analyses of T2DM, MetS, CHD, and stroke, respectively.

\section{White rice consumption and type 2 diabetes}

After pooling data from eight studies, ${ }^{5-820222328}$ we did not find an increased risk of developing T2DM associated with white rice consumption (pooled RR 1.08, 95\% CI 0.87 to 1.33 ; $\mathrm{p}=0.33$ ). Additional stratified analyses across a number of key study characteristics, including region, the quality of included studies, exposure assessment, type of study, and diagnostic criteria, were also performed to explore whether associations with white rice consumption and T2DM differ from those key study characteristics and to confirm the consistency of our primary results (figure 3). In addition, a subgroup analysis of geographical location showed a non-significant association between T2DM among separate Asian and Western populations (eFigure 1). Overall, no association between the risk of developing T2DM and white rice consumption was consistently found in stratified analyses.

\section{White rice consumption and metabolic syndrome}

The pooled analysis of five studies ${ }^{14-17} 2122$ yielded a significant $44 \%$ increase in risk of MetS (RR 1.44, 95\% CI 1.10 to 1.90 ; $\mathrm{p}=0.02)$ with substantial heterogeneity $\left(I^{2}=65.5 \%, \mathrm{p}=0.01\right)$. Subgroup analyses by region showed that there was a statistically significant association between white rice consumption and MetS risk in Iranian populations vs non-Iranian populations (pooled RR 1.47 vs 1.26 , P interaction=0.001). With subgroup analyses, our results demonstrated that there was no statistically significant difference between diagnostic criteria by NCEP-ATP III and modified NCEP-ATP III.

\section{White rice consumption and coronary heart disease and stroke}

After pooling data from four studies ${ }^{14-17}$ for CHD and four studies $^{14} 1^{17-19}$ for stroke, our results showed that white rice consumption was not associated with CHD $(p=0.91)$ or stroke $(\mathrm{p}=0.58)$.

\section{Subgroup analysis}

To determine the effect that differences between subgroups had on pooled estimates, we stratified results by age, sex, diagnostic 


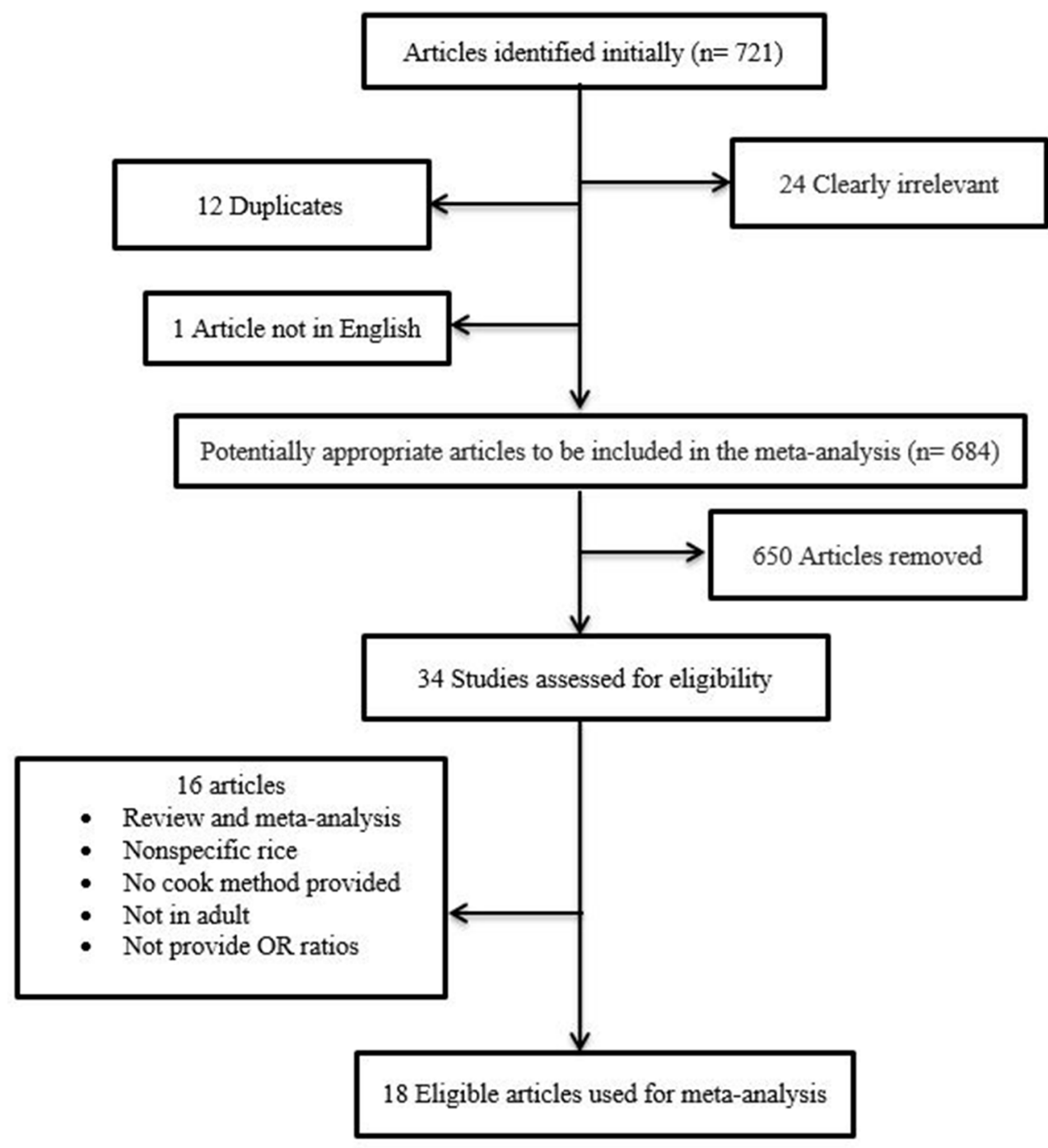

Figure 1 Study design. Flow chart illustrating the selection process for published reports.

criteria, follow-up years, geographic location, type of study, type of exposure and outcome assessments, and the quality of included studies. Most tests for interaction between subgroups were not statistically significant. In addition, meta-regression was performed; however, there were no statistically significant findings.

\section{Sensitivity analysis}

We did several additional analyses to examine the robustness of exploratory results. To assess whether any one study had a dominant effect on the pooled RR, we performed leave-one-out sensitivity analysis, and its effect on the main summary estimate along with pertinent $I^{2}$ for heterogeneity was evaluated. We found that no single study markedly affected the summary estimate or $\mathrm{p}$ values for heterogeneity in T2DM, MetS, CHD, and stroke (eTable 5).

\section{DISCUSSION}

To our knowledge, this is the first meta-analysis of a large number of non-randomised studies to investigate the association between white rice consumption and MetS, CHD, and stroke. Two main findings were forthcoming from the present data: (1) consumption of white rice was not significantly associated with T2DM, CHD and stroke; and (2) high white rice intake was associated with MetS, particularly in Eastern Asian and Iranian populations.

\section{White rice consumption and type 2 diabetes}

Interestingly, in a pooled analysis of three prospective studies published in 2012, $\mathrm{Hu}$ and colleagues ${ }^{4}$ concluded that higher consumption of white rice may be associated with a significantly increased risk of T2DM, especially in Asian populations. Similarly, Sun $e t a l^{5}$ reported that replacement of white rice by brown rice may be associated with a lower risk of T2DM. However, a recent cross-sectional study ${ }^{6}$ among Chinese adults reported an inverse association between T2DM and high white rice consumption. In addition, another prospective study with a 6-year follow-up, conducted by Soriguer et $a l^{7}$ reported that there was an inverse association between white rice consumption and the incidence of T2DM in Southern Spain. We did several stratified and sensitivity analyses to confirm the consistency of the primary results. Overall, our meta-analysis found no association between high white rice consumption and the risk of developing T2DM.

\section{White rice consumption and metabolic syndrome}

According to the results presented here, white rice consumption was shown to be associated with a significantly higher prevalence and/or incidence of MetS with an overall summary RR 
Table 1 Characteristics of included studies

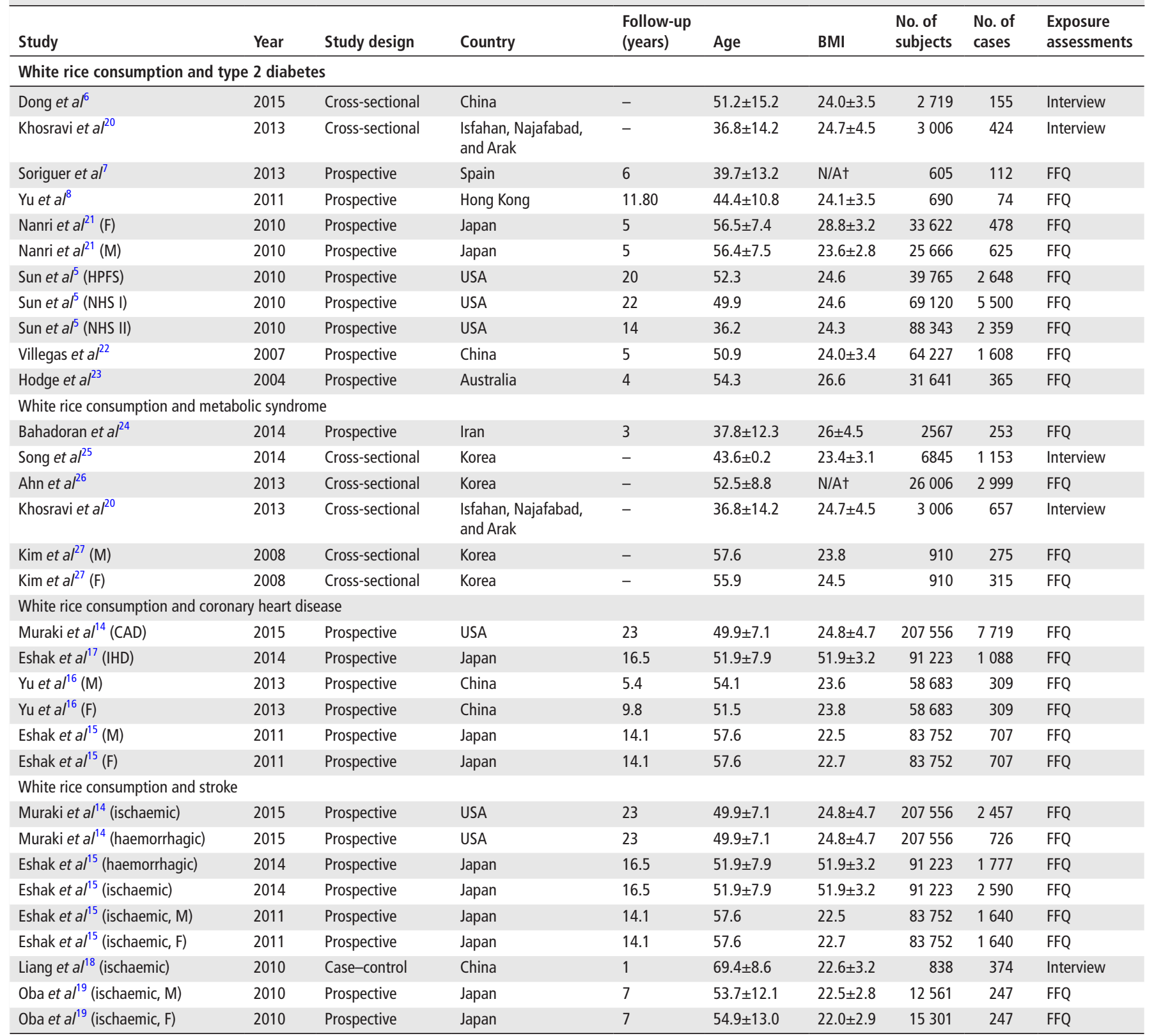

BMI, body mass index; CAD, coronary artery disease; F, female; FFQ, food frequency questionnaires; HPFS, the Health Professionals Follow-up Study; IHD, ischaemic heart disease; M, male; NHS I, Nurses' Health Study I; NHS II, Nurses' Health Study II.

of 1.30. The molecular mechanism underlying the high dietary glycaemic index (ie, white rice-induced MetS) remains unclear, but possible explanations have included excessive compensatory hyperinsulinaemia, lipogenesis (positively regulated by insulin), and insulin resistance states which are the hallmarks of MetS pathogenesis. $^{29}$

Furthermore, the diagnostic criteria of MetS are still in flux among various guidelines. These various definitions from the WHO, NCEP-ATP III and IDF criteria might influence the difference in the incidence and/or prevalence of MetS cases. For example, previous studies ${ }^{30}$ showed that $32-34 \%$ all US adults have MetS using the NCEP-ATP III criteria, whereas such estimates were put at $39 \%$ using the IDF criteria. However, on sensitivity analyses by diagnostic criteria, we found that included studies, evaluated using the NCEP-ATP III criteria, were not associated with the incidence of MetS, compared with modified NCEP-ATP III or other criteria. In fact, none of included studies reported the diagnostic criteria of the MetS using IDF criteria.

The present meta-analysis is consistent with a statistically significant correlation between white rice consumption (high glycaemic index) and MetS perhaps due to geographical distribution differences and varying diagnostic criteria. However, more studies with larger sample sizes and longer durations of follow-up are necessary to explore whether racial differences could yield different results across ethnic groups.

\section{Comparison with other studies}

To our knowledge, no previous meta-analysis has investigated the relationship between white rice consumption and the risk of $\mathrm{CHD}$, stroke and MetS. Contrasting evidence provided by the results of previous meta-analyses of four prospective cohorts of 


\begin{tabular}{|c|c|}
\hline Muraki et al, 2015 & $0.84(0.69-$ \\
\hline Eshak et al, 2014 & $1.08(0.84-$ \\
\hline Yu et al $(M), 2013$ & $2.01(0.96$ \\
\hline Yu et al $(F), 2013$ & $1.53(0.64-$ \\
\hline Eshak et al (M), 2011 & $0.70(0.49-$ \\
\hline Eshak et al (F), 2011 & $1.08(0.66-$ \\
\hline Subtotal $F^{2}=53.2 \%,(P=.06)$ & $0.99(0.79-$ \\
\hline \multicolumn{2}{|c|}{ White Rice Consumption and Stroke } \\
\hline $\begin{array}{l}\text { Muraki et al, } 2015 \\
\text { Eshak et al (Hemorrhagic), } 2014\end{array}$ & $\begin{array}{l}0.84(0.69-1.02) \\
0.96(0.79-1.15)\end{array}$ \\
\hline Eshak et al (Ischemic), 2014 & $1.05(0.90-1.22)$ \\
\hline Eshak et al (M), 2011 & $0.97(0.90-1.04)$ \\
\hline $\begin{array}{l}\text { Eshak et al }(\mathrm{F}), 2011 \\
\text { Liang et al, } 2010\end{array}$ & $\begin{array}{l}0.97(0.89-1.06) \\
2.73(1.31-5.69)\end{array}$ \\
\hline Oba et al (M), 2010 & $0.84(0.43-1.62)$ \\
\hline Oba et al (F), 2010 & $1.37(0.64-2.94)$ \\
\hline Subtotal $F^{2}=80.8 \%,(P<.001)$ & $0.98(0.90-1.06)$ \\
\hline
\end{tabular}
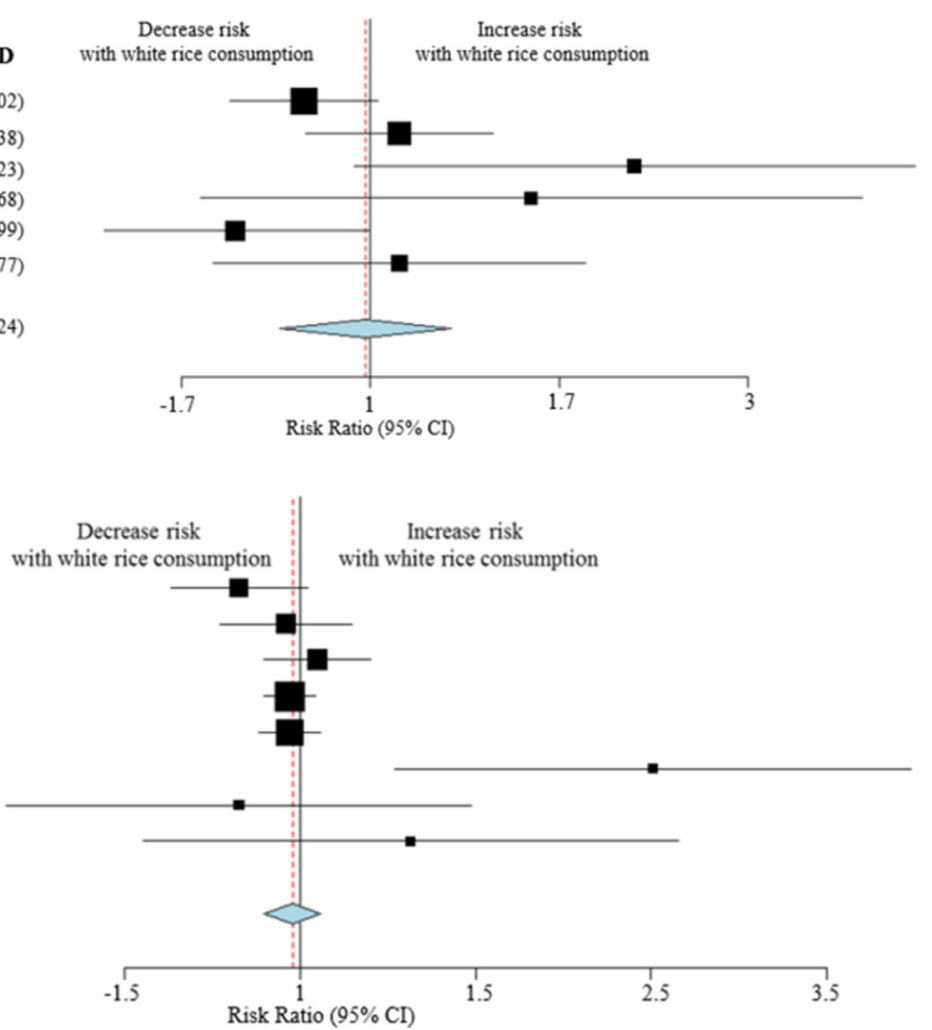

Figure 2 White rice consumption and coronary heart disease (CHD) and stroke. Forest plot of point estimates and confidence intervals also includes results for variance, used in the inverse variance correction.

13284 cases of $\mathrm{T}^{2} \mathrm{DM}^{4}$ have implied that higher consumption of white rice was associated with a significantly increased risk of T2DM (RR $1.11,95 \%$ CI 1.08 to $1.14 ; \mathrm{p}<0.001$ ), especially in Asian (Chinese and Japanese) populations. Interestingly, our present meta-analysis yielded different results which did not support an association between T2DM and white rice consumption. This might be because we have included all recent studies regardless of study design. Stratified analyses also did not yield any

\begin{tabular}{ll}
\multicolumn{2}{c}{ White Rice Consumption and MetS } \\
Bahadoran et al, 2014 & $1.66(1.04-2.66)$ \\
Song et al, 2014 & $1.74(1.23-2.48)$ \\
Ahn et al, 2013 & $1.03(0.83-1.28)$ \\
Khosravi et al, 2013 & $1.25(0.72-2.18)$ \\
Kim et al $(\mathrm{M}), 2008$ & $2.11(0.77-5.75)$ \\
Kim et al(F), 2008 & $2.05(0.92-4.56)$ \\
Subtotal $I^{2}=65.5 \%,(P=.01)$ & $1.44(1.10-1.90)$
\end{tabular}

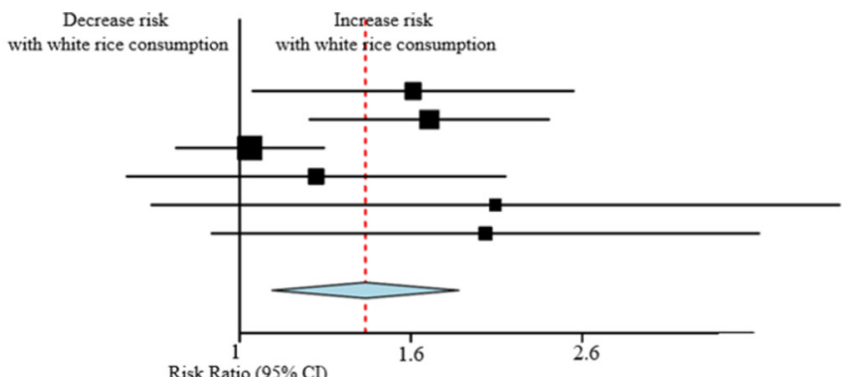

White Rice Consumption and T2DM

$\begin{array}{ll}\text { Dong et al, 2015 } & 0.59(0.36-0.99) \\ \text { Khosravi et al, 2013 } & 1.06(0.47-2.43) \\ \text { Soriguer et al, 2013 } & 0.43(0.19-0.95) \\ \text { Yu et al, 2011 } & 0.87(0.78-1.34) \\ \text { Nanri et al (W), 2010 } & 1.65(1.06-2.57) \\ \text { Nanri et al (M), 2010 } & 1.19(0.85-1.68) \\ \text { Sun et al (HPFS), 2010 } & 1.02(0.77-1.34) \\ \text { Sun et al (NHS I), 2010 } & 1.11(0.87-1.43) \\ \text { Sun (NHS II), 2010 } & 1.40(1.09-1.80) \\ \text { Villegas et al, 2007 } & 1.78(1.48-2.15) \\ \text { Hodge et al, 2004 } & 0.89(0.66-1.21) \\ & \\ \text { Subtotal } I^{2}=80.8 \%,(\mathrm{P}<.001) & 1.08(0.87-1.33)\end{array}$

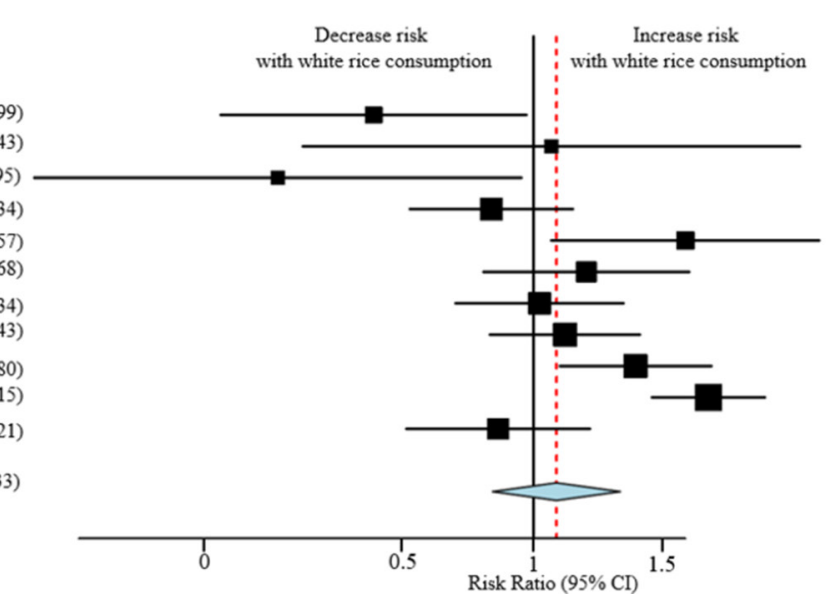

Figure 3 White rice consumption and metabolic syndrome (MetS) and type 2 diabetes mellitus (T2DM). Forest plot of point estimates and confidence intervals also includes results for variance, used in the inverse variance correction. 
statistically significant findings. More recently, some studies ${ }^{6-8}$ found an inverse relationship between white rice consumption and T2DM. However, another recent study ${ }^{20}$ of white rice consumption found a small positive trend to an increased risk of T2DM. Sensitivity analyses, including the quality of included studies, type of study design, geographical location, exposure/ outcome assessments, and leave-one-out method, indicated that there was no statistically significant association between white rice consumption and T2DM.

\section{Strengths and limitations}

The present meta-analysis has several strengths. First, the large sample size and number of studies included, regardless of study design, facilitate a robust and accurate analysis of the effects of any single study. Second, the success in reaching data from most study authors overcame the lack of key data in previous published reports. Third, this meta-analysis was designed to be highly comprehensive with a robust search strategy along with a manual search for web science and unpublished studies.

However, our study also has certain limitations and our results should be interpreted with caution. First, a lack of adjustment for possible confounders (genetics, gene-diet interaction, traditional rice-eating pattern) could produce a superficially strong association between high white rice consumption and the prevalence of MetS in certain populations. Second, the cooking method and accompanying ingredients were not available in most of the included studies, and definitions for high or low white rice intake were not standardised. Third, during the long follow-up, participants may have changed their diets and rice-eating behaviours. Fourth, most included studies are non-experimental studies, and this might lead to selection bias. Fifth, international standards of definition for white rice consumption vary across different populations and locations. Sixth, heterogeneity introduced by including diverse disease outcomes and study designs is also a limitation. Seventh, we contacted all relevant authors for further information about types of stroke (ischaemic vs haemorrhagic), but, unfortunately, those included studies had limited power to evaluate the stroke types. Eighth, cross-sectional studies of self-reported diet and retrospective case-control studies have a very high likelihood of bias. Lastly, although we did not find publication bias, there might be limited power to detect such a bias.

\section{Key messages}

What is already known about this subject?

The current Dietary Guidelines for Americans 2015-2020 advocate replacing refined grains (eg, white rice) with whole grains (eg, brown rice) for improved health outcomes. However, less is known about the metabolic and cardiovascular outcomes associated with white rice consumption.

What does this study add?

Our meta-analysis did not find an association between white rice consumption and the risk of developing type 2 diabetes mellitus, coronary heart disease or stroke. However, high white rice consumption may be associated with an increased risk of metabolic syndrome in some populations.

How might this impact on clinical practice? Until evidence from large long-term prospective studies confirm these findings, nutritional counselling should reinforce the consumption of whole grains.

\section{CONCLUSIONS}

In summary, our meta-analysis did not find an association between white rice consumption and risk of developing T2DM, CHD or stroke. These findings may provide reassurance as to the safety of white rice consumption when counselling patients. However, white rice consumption did not demonstrate a protective effect in relation to cardiovascular outcomes. In addition, high white rice intake was associated with MetS, particularly in Iranian populations. The results from the present study should be interpreted in light of the observational study design, difficulty in quantifying the exposure, and the problem of heterogeneity due to varying methods for measuring rice consumption. More studies with larger sample sizes in diverse geographical locations and with longer follow-up periods are needed to examine racial differences. Therefore, until evidence from large long-term prospective studies confirm our findings, nutritional counselling should reinforce the consumption of whole grains.

Acknowledgements The authors thank Kimberly J Bisanz, RDN, LD (Department of Diet-Clinical Nutrition, Mayo Clinic, Minnesota) for nutitional consultations and Molly M McMahon, MD (Department of Endocrinology, Diabetes, Metabolism and Nutrition, Mayo Clinic, Minnesota) for endocrinology consultations. The authors also thank Danxia Yu, PhD (Vanderbilt University), Qi Sun, DSc, MD (Brigham and Women's Hospital and Harvard School of Public Health), Isao Muraki, MD, PhD (Osaka Center for Cancer and Cardiovascular Disease Prevention), Fei Dong, PhD (University of North Carolina at Chapel Hill), Penny Gordon-Larsen, PhD, FAHA, FTOS, (Carolina Population Center and University of North Carolina), Raquel Villegas, PhD, Xiao Ou Shu, MD, PhD, (Vanderbilt Epidemiology Center, VanderbiltUniversity Medical Center), Andy H Lee, PhD, Wenbin Liang, MBBS, MPH, PhD (CurtinUniversity of Technology), Allison M. Hodge, MENVSC (Cancer Epidemiology Centre and TheCancer Council Victoria), YoonJu Song, PhD (The Catholic University of Korea), Shino Oba, PhD, MSPH (Kanagawa University of Human Services), Jihye Kim, PhD, Inho Jo, PhD (Graduate School of East-West Medical Science, Kyung Hee University), Koh Woon-Puay, PhD (Duke-NUS Medical School, Singapore) and Hossein KhosraviBoroujeni, PhD (School of Medicine and Menzies Health Institute, Griffith University) for providing additional information about their studies.

Contributors CK, AT, SC, HZ, ZS, LJP, HW had full access to all of the data in the study and take responsibility for the integrity of the data and the accuracy of the data analysis. Study concept and design:CK, AT,HW. Acquisition of data: CK, LJP. Analysis and interpretation of data: CK, ZW, TS.Drafting of the manuscript: CK and ZW. Critical revision of the manuscript for important intellectual content: $\mathrm{SCh}, \mathrm{HZ}$, TS. Study supervision: CK and TS.

Competing interests All authors have completed and submitted the ICMJE Form for Disclosure of Potential Conflicts of Interest and none were reported.

Patient consent This is not a human subject study.

Provenance and peer review Not commissioned; externally peer reviewed.

(c) Article author(s) (or their employer(s) unless otherwise stated in the text of the article) 2017. All rights reserved. No commercial use is permitted unless otherwise expressly granted.

\section{REFERENCES}

1 US Department of Health and Human Services. US Department of Agriculture. 2015 2020 dietary guidelines for Americans. 8th ed. Washington, DC, 2015. Avaliable online at. http://www.health.gov/DietaryGuidelines.

2 Shimabukuro M, Higa M, Kinjo R, et al. Effects of the brown rice diet on visceral obesity and endothelial function: the BRAVO study. Br J Nutr 2014;111:310-20.

3 Mohan V, Spiegelman D, Sudha V, et al. Effect of brown rice, white rice, and brown rice with legumes on blood glucose and insulin responses in overweight Asian Indians: a randomized controlled trial. Diabetes Technol Ther 2014;16:317-25.

4 Hu EA, Pan A, Malik V, et al. White rice consumption and risk of type 2 diabetes: meta-analysis and systematic review. BMJ 2012;344:e1454.

5 Sun Q, Spiegelman D, van Dam RM, et al. White rice, brown rice, and risk of type 2 diabetes in US men and women. Arch Intern Med 2010;170:961-9.

6 Dong $F$, Howard $A G$, Herring $A H$, et al. White rice intake varies in its association with metabolic markers of diabetes and dyslipidemia across region among Chinese adults. Ann Nutr Metab 2015:66:209-18.

7 Soriguer F, Colomo N, Olveira G, et al. White rice consumption and risk of type 2 diabetes. Clin Nutr 2013;32:481-4. 
8 Yu R, Woo J, Chan R, et al. Relationship between dietary intake and the development of type 2 diabetes in a Chinese population: the Hong Kong Dietary Survey. Public Health Nutr 2011;14:1133-41.

9 Aune D, Keum N, Giovannucci E, et al. Whole grain consumption and risk of cardiovascular disease, cancer, and all cause and cause specific mortality: systematic review and dose-response meta-analysis of prospective studies. BMJ 2016;353:i2716.

10 Stang A. Critical evaluation of the Newcastle-Ottawa scale for the assessment of the quality of nonrandomized studies in meta-analyses. Eur J Epidemiol 2010;25:603-5.

11 DerSimonian R, Laird N. Meta-analysis in clinical trials. Control Clin Trials 1986:7:177-88.

12 Mantel N, Haenszel W. Statistical aspects of the analysis of data from retrospective studies of disease. J Natl Cancer Inst 1959;22:719-48.

13 Stroup DF, Berlin JA, Morton SC, et al. Meta-analysis of observational studies in epidemiology: a proposal for reporting. Meta-analysis of Observational Studies in Epidemiology (MOOSE) group. JAMA 2000;283:2008-12.

14 Muraki I, Wu H, Imamura F, et al. Rice consumption and risk of cardiovascular disease: results from a pooled analysis of 3 U.S. cohorts. Am J Clin Nutr 2015;101:164-72.

15 Eshak ES, Iso H, Yamagishi K, et al. Rice consumption is not associated with risk of cardiovascular disease morbidity or mortality in Japanese men and women: a large population-based, prospective cohort study. Am J Clin Nutr 2014;100:199-207.

16 Yu D, Shu XO, Li H, et al. Dietary carbohydrates, refined grains, glycemic load, and risk of coronary heart disease in Chinese adults. Am J Epidemiol 2013;178:1542-9.

17 Eshak ES, Iso H, Date C, et al. Rice intake is associated with reduced risk of mortality from cardiovascular disease in Japanese men but not women. J Nutr 2011;141:595-602.

18 Liang W, Lee $\mathrm{AH}$, Binns CW. White rice-based food consumption and ischemic stroke risk: a case-control study in southern China. J Stroke Cerebrovasc Dis 2010;19:480-4.

19 Oba S, Nagata C, Nakamura K, et al. Dietary glycemic index, glycemic load, and intake of carbohydrate and rice in relation to risk of mortality from stroke and its subtypes in Japanese men and women. Metabolism 2010;59:1574-82.
20 Khosravi-Boroujeni H, Sarrafzadegan N, Mohammadifard N, et al. White rice consumption and CVD risk factors among Iranian population. $J$ Health Popul Nutr 2013;31:252-61.

21 Nanri A, Mizoue T, Noda M, et al. Rice intake and type 2 diabetes in Japanese men and women: the Japan Public Health Center-based prospective study. Am J Clin Nutr 2010;92:1468-77.

22 Villegas R, Liu S, Gao YT, et al. Prospective study of dietary carbohydrates, glycemic index, glycemic load, and incidence of type 2 diabetes mellitus in middle-aged Chinese women. Arch Intern Med 2007;167:2310-6.

23 Hodge AM, English DR, O'Dea K, et al. Glycemic index and dietary fiber and the risk of type 2 diabetes. Diabetes Care 2004;27:2701-6.

24 Bahadoran Z, Mirmiran P, Delshad H, et al. White rice consumption is a risk factor for metabolic syndrome in Tehrani adults: a prospective approach in Tehran lipid and glucose study. Arch Iran Med 2014;17:435-40.

25 Song $\mathrm{S}$, Lee JE, Song WO, et al. Carbohydrate intake and refined-grain consumption are associated with metabolic syndrome in the Korean adult population. J Acad Nutr Diet 2014;114:54-62.

26 Ahn Y, Park SJ, Kwack HK, et al. Rice-eating pattern and the risk of metabolic syndrome especially waist circumference in Korean Genome and Epidemiology Study (KoGES). BMC Public Health 2013;13:61.

27 Kim K, Yun SH, Choi BY, et al. Cross-sectional relationship between dietary carbohydrate, glycaemic index, glycaemic load and risk of the metabolic syndrome in a korean population. Br J Nutr 2008;100:576-84.

28 Nanri A, Mizoue T, Yoshida D, et al. Dietary patterns and a1c in Japanese men and women. Diabetes Care 2008;31:1568-73.

29 Biddinger SB, Kahn CR. From mice to men: insights into the insulin resistance syndromes. Annu Rev Physiol 2006;68:123-58.

30 Ford ES, Li C, Zhao G. Prevalence and correlates of metabolic syndrome based on a harmonious definition among adults in the US. J Diabetes 2010;2:180-93. 